\section{Exposição ao aleitamento materno e transtornos mentais comuns na adolescência}

\author{
Exposure to breastfeeding and common mental \\ disorders in adolescence
}

Exposición a la lactancia materna y trastornos
mentales comunes en la adolescencia

\section{Resumo}

O objetivo foi avaliar o efeito da exposição e do tempo de exposição ao aleitamento materno na ocorrência de transtornos mentais comuns (TMC) entre adolescentes escolares brasileiros. Este trabalho analisou dados do Estudo de Riscos Cardiovasculares em Adolescentes (ERICA), avaliando aqueles que tiveram o questionário referente ao aleitamento materno respondido pelos pais ou responsáveis. A presença de TMC foi identificada pelo General Health Questionnaire, versão 12 itens (GHQ-12), considerando-se dois pontos de corte (escores $\geq 3 e \geq 5$ ). As associações foram testadas em análises bivariadas e por meio de modelos de regressão logística múltipla, com ajustes por variáveis potenciais de confusão. Dentre os 41.723 adolescentes avaliados, a maioria foi composta por estudantes do sexo feminino (54,6\%), que tinham idades entre 12 e 15 anos (71\%), estudavam em escolas públicas (83,1\%), residiam na Região Sudeste (51,9\%) e eram das classes econômicas B $(53,8 \%)$ e C $(34,1 \%)$. Cerca de metade das mães dos adolescentes não tinha o ensino médio completo $(51,7 \%)$. O grupo de adolescentes com mais de seis meses de aleitamento materno $(51,8 \%)$ apresentou uma menor prevalência de TMC para os dois pontos de corte do GHQ-12 avaliados, quando comparado com o grupo que não recebeu aleitamento materno ou que o recebeu por período $\leq 1$ mês $(R I=0,82$; IC95\%:0,69-0,97 e $R I=0,74$; IC95\%: 0,59-0,91 para 3 e 5 pontos, respectivamente). $O$ aleitamento materno prolongado parece desempenhar um papel protetor para a ocorrência de TMC na adolescência.

Saúde Mental; Adolescente; Transtornos Mentais; Aleitamento Materno
Caroline Rodrigues de Almeida 1

Evandro Silva Freire Coutinho 2

Daniela Alves Silva 1

Elizabete Regina Araújo de Oliveira 1

Katia Vergetti Bloch 3

Maria Carmen Viana 1

doi: 10.1590/0102-311X00093718

\author{
Correspondência \\ C. R. Almeida \\ Universidade Federal do Espírito Santo. \\ Av. Marechal Campos 1468, Vitória, ES 29047-105, Brasil. \\ c.r.almeida2@hotmail.com \\ 1 Universidade Federal do Espirito Santo, Vitória, Brasil. \\ 2 Escola Nacional de Saúde Pública Sergio Arouca, Fundação \\ Oswaldo Cruz, Rio de Janeiro, Brasil. \\ 3 Universidade Federal do Rio de Janeiro, Rio de Janeiro, Brasil.
}




\section{Introdução}

Em todo o mundo, entre 10 e $20 \%$ das crianças e adolescentes sofrem de transtornos mentais, frequentemente com sintomas persistentes e curso crônico, podendo levar ao comprometimento do bem-estar geral, do desenvolvimento físico e psicológico, e da capacidade intelectiva, impactando o seu desempenho no âmbito pessoal, social, ocupacional e familiar na vida adulta 1,2. As estimativas de prevalência de transtornos mentais em crianças e adolescentes variam em função do sexo, da idade, do tipo de transtorno avaliado e da população estudada, e depende dos instrumentos de avaliação utilizados. Sabe-se que características familiares, sociais, econômicas e psicológicas, assim como a exposição a experiências adversas na infância, são fatores que influenciam o desenvolvimento de problemas de comportamento e de transtornos mentais durante a adolescência 3,4.

Em revisão sistemática de 27 estudos, os transtornos mais frequentemente identificados entre crianças e adolescentes em diferentes países e culturas incluem a depressão (1\% a 30\%), transtornos de ansiedade (3,3\% a 32,3\%), transtorno do déficit de atenção com hiperatividade (TDAH) (0,9\% a 19\%), transtorno por uso de substâncias $(1,7 \%$ a $32,1 \%)$ e transtorno de conduta $(1,8 \%$ a $29,2 \%)$. Um inquérito realizado no Brasil, em 2008, identificou que 12,6\% dos brasileiros de 6 a 17 anos apresentavam algum transtorno mental, o equivalente a 5 milhões de crianças e adolescentes 6,7 .

No entanto, pouco se sabe acerca do impacto do aleitamento materno sobre a saúde mental subsequente. $\mathrm{O}$ ato de amamentar é a primeira ação de prevenção de doenças e intervenção nutricional, empreendida pela mãe para assegurar a sobrevivência e saúde do filho ${ }^{8}$. Os benefícios imediatos e de curto prazo do aleitamento materno têm sido amplamente descritos na literatura médica ${ }^{9}$. Estudos de seguimento têm demonstrado o papel positivo da amamentação prolongada no desempenho em testes cognitivos 10 , de vocabulário e coordenação visuomotora, além de estar associada a medidas adequadas de altura e da circunferência cefálica 11, a uma maior renda na idade adulta 12, e menor ocorrência de problemas de comportamento e transtornos internalizantes 11,13,14,15,16. Diversos autores identificaram o papel protetor da amamentação na redução de estresse psicológico, sintomas de depressão/ansiedade, transtorno do déficit de atenção e depressão maior em crianças e adolescentes $14,17,18,19,20,21,22,23$. Outros estudos, no entanto, não identificaram associações significativas entre aleitamento materno e transtornos mentais $24,25,26,27$.

A principal hipótese deste trabalho é que o aleitamento materno por um período mais prolongado atue como fator de proteção para o desenvolvimento de transtornos mentais comuns (TMC) na adolescência, que se caracterizam pela ocorrência de sintomas depressivos, ansiosos e somáticos, sem alterações psicóticas 28 . Assim, este estudo teve como objetivo avaliar o efeito da exposição e do tempo de exposição ao aleitamento materno na ocorrência de TMC entre adolescentes escolares brasileiros.

\section{Métodos}

Este trabalho analisou os dados do Estudo de Riscos Cardiovasculares em Adolescentes (ERICA), um estudo transversal, nacional, de base escolar, conduzido no biênio 2013-2014, que avaliou uma amostra representativa de adolescentes de 12-17 anos de idades, em municípios brasileiros com mais de 100 mil habitantes em cada macrorregião geográfica do país, totalizando 74.589 alunos 29 . Teve por objetivos estimar a prevalência de síndrome metabólica, diabetes mellitus, obesidade, fatores de risco cardiovascular e de marcadores de resistência à insulina e inflamatórios 30. Foi usado um coletor de dados eletrônico, preenchido pelo próprio adolescente no âmbito da escola.

Informações sobre morbidade familiar, peso ao nascer do adolescente e histórico de amamentação foram obtidas por meio de questionário enviado pelos alunos aos pais ou responsáveis ${ }^{30}$. Para o presente estudo, foram incluídos todos os adolescentes cujos pais ou responsáveis responderam ao questionário que continha informações sobre o aleitamento materno recebido pelo adolescente ( $\mathrm{n}=41.723 ; 55,9 \%$ da amostra total). Em uma análise prévia de sensibilidade, verificou-se que não há diferença significativa na prevalência de TMC entre os alunos cujos pais não responderam e os que responderam ao questionário com perguntas sobre amamentação (TMC3: $\chi^{2}=18,7 ; \mathrm{p}=0,11$; TMC5: $\left.\chi^{2}=0,43 ; p=0,77\right)$. Foi investigado se a criança foi amamentada no peito e durante quanto tempo 
após o nascimento. A exposição ao aleitamento materno foi, então, categorizada em três grupos: (1) não recebeu aleitamento materno/ou recebeu por período inferior ou igual a um mês; (2) recebeu aleitamento materno de mais de 1 mês até seis meses; e (3) recebeu aleitamento materno por período superior a seis meses.

O desfecho analisado foi a presença de TMC, avaliada por meio do General Health Questionnaire, versão 12 itens (GHQ-12), um instrumento de rastreamento para TMC, desenvolvido por Goldberg em 1972, em sua versão original com 60 itens, tendo sido criadas versões reduzidas com base em estudos com análises fatoriais, tornando-o uma ferramenta útil em contextos que requerem avaliações rápidas sobre desconforto psicológico sem comprometer evidências de confiabilidade de suas pontuações 28,31 . Os itens individuais foram codificados como "ausente" ou "presente" (0 ou 1, respectivamente) e, então, somados em um total possível de 12 pontos. Neste estudo foram utilizados dois pontos de corte do GHQ-12: valores iguais ou maiores que 3 (TMC3), que é o ponto de corte usado em publicação anterior ao ERICA 32; e valores iguais ou maiores que 5 (TMC5), que identificam possíveis casos com sintomatologia mais intensa/frequente, com maiores índices de especificidade 28,31 . Vale ressaltar que se trata de um instrumento de rastreamento de TMC, uma vez que o padrão-ouro para o diagnóstico é a entrevista psiquiátrica padronizada 28. O alfa de Cronbach foi de 0,86, mostrando boa consistência interna do GHQ-12 neste trabalho.

Inicialmente foram calculadas as proporções de aleitamento materno $(\leq 1$ mês; $1-6$ meses; $>6$ meses) segundo variáveis sociodemográficas (sexo, idade, cor da pele do adolescente, classe econômica, escolaridade materna, tipo de escola e região de residência). A classe econômica foi definida com base no Critério de Classificação Econômica do Brasil, desenvolvido pela Associação Brasileira de Empresas de Pesquisas (ABEP), que considera a posse de itens e grau de escolaridade do chefe da família na atribuição da pontuação (A: 35-46 pontos; B: 23-34 pontos; C: $14-22$ pontos; D/E: menor que 14) 33 .

Em seguida, estimou-se a proporção de adolescentes com TMC, segundo os dois pontos de corte (TMC3 e TMC5), de acordo com o tempo de aleitamento. Esses dados foram calculados e estratificados segundo as variáveis sociodemográficas. Com o intuito de avaliar o impacto das perdas sobre nossos resultados, foram calculadas as proporções de TMC3 e TMC5, assim como de covariáveis de interesse, entre os adolescentes com e sem informação sobre a exposição ao aleitamento materno.

Modelos de regressão logística simples e múltipla foram ajustados por variáveis de confundimento, para estimar a associação entre aleitamento e TMC por meio da razão de taxas de incidência. Como demonstrado por Pearce 34 e Reichenheim \& Coutinho 35, a exponencial do coeficiente de regressão logística pode estimar a taxa de densidade de incidência quando as seguintes condições são atendidas: (i) A população se mantém estável durante o período de estudo (estado estacionário); (ii) ausência de sobrevivência seletiva; (iii) a exposição não influencia a duração do desfecho; (iv) ausência de causalidade reversa. As variáveis para ajuste de confundimento foram definidas em função do padrão de associação com a exposição e com o desfecho identificado com base na literatura e nas análises exploratórias, e foram testadas, inicialmente, uma a uma.

Pela natureza complexa do delineamento da amostra, foram utilizados pesos amostrais em todas as análises, calculados pelo produto dos inversos das probabilidades de inclusão em cada estrato da amostra e calibrados considerando-se uma estimativa da população de adolescentes matriculados em escolas localizadas nos estratos geográficos, levando-se em consideração a distribuição por sexo e idade. O detalhamento dos métodos de amostragem e ponderação foram descritos por Vasconcelos et al. 36 .

Avaliou-se o potencial de interação das variáveis sexo e idade na associação entre a exposição e o desfecho por meio da inclusão de termos de interação no modelo. Visto que não houve modificação de efeito, os resultados não foram estratificados por essas variáveis.

Todas as análises foram realizadas usando-se o software Stata versão 15 (https://www.stata.com), com nível de $5 \%$ de significância para todos os testes.

O ERICA foi aprovado pelos Comitês de Ética em Pesquisa do Instituto de Estudos de Saúde Coletiva da Universidade Federal do Rio de Janeiro, instituição da Coordenação Central do estudo (IESC/ UFRJ - Processo 45/2008), e de uma instituição de Ensino Superior em cada estado brasileiro 30. 


\section{Resultados}

Dentre os adolescentes incluídos neste estudo ( $n=41.723)$, a maioria foi composta por estudantes do sexo feminino (54,6\%) e com idades entre 12 e 15 anos (71\%). Mais de $80 \%$ estudavam em escolas públicas $(83,1 \%)$, cerca de metade residia na Região Sudeste $(51,9 \%)$ e era da classe econômica B $(53,8 \%)$, seguida pela classe C $(34,1 \%)$. Aproximadamente a metade das mães dos adolescentes não completou o Ensino Médio (51,7\%) (Tabela 1).

A maioria dos adolescentes recebeu aleitamento materno por um período superior a seis meses (51,8\%; IC95\%: 50,7-52,9), já 14,9\% (IC95\%: 14,1-15,7) e 33,3\% (IC95\%: 32,1-34,6) foram amamentados por $\leq 1$ mês e de 1 a 6 meses, respectivamente (Tabela 1 ).

Ainda de acordo com a Tabela 1, não houve diferença no padrão de aleitamento materno por sexo ou idade dos adolescentes ( $\mathrm{p}=0,52 \mathrm{e} \mathrm{p}=0,49$, respectivamente). Quanto à cor da pele, há uma tendência que sugere que adolescentes de cor amarela sejam aqueles com menor proporção de amamentação $\leq 1$ mês $(10,6 \%)$, e os de cor branca sejam aqueles com menor proporção de amamentação mais prolongada $(49,8 \% ; \mathrm{p}=0,07)$. Adolescentes das classes econômicas D e E apresentaram maior frequência de aleitamento materno ausente ou por um período curto ( $\leq 1$ mês) e menor frequência de aleitamento materno prolongado ( $>6$ meses; $\mathrm{p}=0,004$ ). Além disso, observou-se que quanto menor o grau de escolaridade da mãe, maior era a proporção de adolescentes com período de aleitamento materno $\leq 1$ mês $(\mathrm{p}<0,001)$. A proporção de adolescentes que foram amamentados por um período $\leq 1$ mês também foi maior entre estudantes das escolas públicas do que das privadas (15,3\% vs. 13\%; $\mathrm{p}<0,001)$. Quanto à macrorregião, adolescentes das regiões Sudeste e Sul $(16,1 \%$ e $16,9 \%$, respectivamente) foram aqueles com maior proporção de exposição ao aleitamento por período $\leq 1$ mês, e proporções maiores daqueles que residiam nas regiões Norte e Centro-oeste receberam aleitamento materno por um período prolongado $(66,1 \%$ e $60,6 \%$, respectivamente; $p<0,001)$.

As prevalências de TMC baseadas nos dois pontos de corte do GHQ estão na Tabela 2, estratificadas pelas características sociodemográficas da amostra. Adolescentes do sexo feminino apresentaram maior prevalência de TMC3 e TMC5, em comparação aos do sexo masculino, independentemente do tempo de amamentação (valor de $\mathrm{p}<0,001$ ). Houve maior prevalência de TMC3 na faixa etária de 15-17 anos, que se manteve independente das categorias de aleitamento materno (valor de $\mathrm{p}<0,001$ ). As diferenças observadas para cor da pele não apresentaram significância estatística em nenhuma das categorias de aleitamento (valor de $\mathrm{p}>0,20$ ). Observou-se prevalências mais elevadas de TMC3 entre estudantes das classes sociais $\mathrm{D} / \mathrm{E}$, entre aqueles com aleitamento materno ausente ou de baixa duração ( $\leq 1$ mês) e entre estudantes da classe A no grupo de amamentação prolongada (valor de $\mathrm{p}<$ $0,05)$. As prevalências de TMC3 não apresentaram diferenças estatisticamente significativas para os graus de escolaridade materna em nenhuma das categorias de aleitamento (valor de $\mathrm{p}>0,10$ ). Quanto à macrorregião, houve menor prevalência de TMC3 na Região Sudeste, com resultados estatisticamente significativos nas categorias de amamentação $\leq 1$ mês e acima de 6 meses valor de $p<0,05$ ). Os achados para TMC5 apresentaram diferenças menos marcantes do que os encontrados para TMC3 (Tabela 2).

A informação sobre o aleitamento materno utilizada nas análises conduzidas neste estudo foi disponibilizada por apenas $56,7 \%$ dos pais ou responsáveis dos adolescentes que compuseram a amostra total do ERICA. Com o intuito de nos assegurarmos da representatividade da subamostra estudada, decidimos investigar a distribuição de TMC (desfecho de interesse) entre os dois grupos. Na Tabela 3 observamos que a prevalência de TMC foi semelhante entre adolescentes incluídos e excluídos das análises, para os dois pontos de corte estudados. Embora essa seja a principal variável do trabalho, também repetimos o processo para variáveis utilizadas no ajuste dos modelos. Não foram observadas diferenças relevantes para cor da pele, macrorregião de residência e escolaridade materna entre aqueles com e sem informação sobre o aleitamento materno (Tabela 3).

$\mathrm{Na}$ Tabela 4, encontram-se as estimativas para a associação entre aleitamento materno e TMC, com medidas brutas e ajustadas individualmente pelas variáveis sociodemográficas. De modo geral, tanto para TMC3 quanto para TMC5, houve uma redução na ocorrência de TMC entre os adolescentes com aleitamento materno superior a seis meses. Essa redução foi de $17 \%$ $(\mathrm{RI}=0,83$; IC95\%: 0,72-0,96) quanto se utilizou o ponto de corte 3 no GHQ-12, e de $23 \%$ (RI = 0,77; IC95\%: 0,64-0,93) para o ponto de corte 5, variando de $17 \%-18 \%$ a $22-26 \%$, respectivamente. 
Tabela 1

Caracterização da amostra de acordo com as características socioeconômicas, demográficas e geográficas e o tempo de aleitamento materno, entre escolares brasileiros de 12-17 anos. Estudo de Riscos Cardiovasculares em Adolescentes (ERICA), 2013-2014 ( $n=41.723)$.

\begin{tabular}{|c|c|c|c|c|}
\hline & \multicolumn{4}{|c|}{ Aleitamento materno (meses) } \\
\hline & Não ou $\leq 1$ & $2-6$ & $>6$ & Total \\
\hline & $\%$ (IC95\%) & $\%($ IC95\%) & $\%(I C 95 \%)$ & $\%($ IC95\%) \\
\hline \multicolumn{5}{|l|}{ Sexo } \\
\hline Feminino & $15,0(13,8-16,4)$ & $32,9(31,0-34,8)$ & $52,1(50,5-53,6)$ & $54,6(53,7-55,4)$ \\
\hline Masculino & $14,7(13,7-15,8)$ & $33,8(32,4-35,2)$ & $51,5(50,0-53,0)$ & $45,4(44,6-46,3)$ \\
\hline \multicolumn{5}{|l|}{ Idade (anos) } \\
\hline 12 & $14,6(13,2-16,0)$ & $32,5(28,5-36,7)$ & $52,9(48,9-56,9)$ & $18,3(17,2-19,4)$ \\
\hline 13 & $14,9(13,1-16,8)$ & $34,3(31,5-37,2)$ & $50,8(48,4-53,2)$ & $18,0(17,2-18,8)$ \\
\hline 14 & $14,1(12,6-15,8)$ & $34,6(32,7-36,6)$ & $51,3(48,6-54,0)$ & $17,4(16,4-18,4)$ \\
\hline 15 & $15,8(14,3-17,3)$ & $31,4(29,6-33,2)$ & $52,8(50,5-55,2)$ & $17,3(16,6-18,0)$ \\
\hline 16 & $15,5(13,4-17,9)$ & $32,6(30,1-35,2)$ & $51,9(50,2-53,7)$ & $15,7(14,8-16,7)$ \\
\hline 17 & $14,5(12,7-16,5)$ & $34,8(32,2-37,5)$ & $50,7(47,7-53,7)$ & $13,3(12,4-14,3)$ \\
\hline \multicolumn{5}{|l|}{ Cor da pele * } \\
\hline Branca & $15,8(14,3-17,4)$ & $34,4(31,8-37,0)$ & $49,8(47,8-51,8)$ & $40,1(38,1-42,2)$ \\
\hline Preta & $14,8(11,7-18,5)$ & $33,2(30,0-36,7)$ & $52,0(47,3-56,6)$ & $7,5(6,8-8,3)$ \\
\hline Parda & $14,3(13,3-15,2)$ & $32,4(30,8-34,0)$ & $53,3(51,8-54,9)$ & $49,7(47,7-51,7)$ \\
\hline Amarela & $10,6(8,2-13,6)$ & $35,7(30,3-41,5)$ & $53,7(48,1-59,2)$ & $2,0(1,7-2,3)$ \\
\hline Indígena & $17,5(10,2-28,5)$ & $28,6(18,6-41,3)$ & $53,8(38,6-68,3)$ & $0,6(0,5-0,8)$ \\
\hline \multicolumn{5}{|l|}{ Classe econômica * } \\
\hline A & $12,4(10,3-14,9)$ & $39,7(36,2-43,4)$ & $47,8(43,8-51,9)$ & $10,4(8,7-12,3)$ \\
\hline B & $14,8(13,4-16,2)$ & $33,6(31,3-36,0)$ & $51,6(49,6-53,6)$ & $53,8(52,3-55,3)$ \\
\hline C & $14,4(12,7-16,3)$ & $32,4(30,2-34,7)$ & $53,2(51,2-55,1)$ & $34,1(32,3-36,0)$ \\
\hline $\mathrm{D} / \mathrm{E}$ & $19,3(11,2-31,1)$ & $38,6(27,4-51,2)$ & $42,1(33,3-51,6)$ & $1,7(1,4-2,1)$ \\
\hline \multicolumn{5}{|l|}{ Escolaridade materna (anos) * } \\
\hline$\geq 0$ e $<4$ & $20,3(17,6-23,2)$ & $29,8(26,3-33,5)$ & $50,0(45,7-54,2)$ & $13,5(11,7-15,4)$ \\
\hline$\geq 4 \mathrm{e}<8$ & $17,2(15,4-19,3)$ & $32,6(30,0-35,2)$ & $50,2(47,6-52,8)$ & $17,6(16,1-19,2)$ \\
\hline$\geq 8 \mathrm{e}<11$ & $13,7(11,8-16,0)$ & $33,7(31,5-36,0)$ & $52,6(50,0-55,1)$ & $20,7(19,6-21,8)$ \\
\hline Ensino Médio completo & $12,1(11,0-13,3)$ & $35,9(34,1-37,8)$ & $52,0(50,2-53,7)$ & $34,5(32,4-36,6)$ \\
\hline Ensino Superior completo & $12,1(10,6-13,9)$ & $37,8(35,4-40,3)$ & $50,0(47,6-52,5)$ & $13,8(11,8-16,0)$ \\
\hline \multicolumn{5}{|l|}{ Tipo de escola * } \\
\hline Pública & $15,3(14,3-16,3)$ & $32,5(31,1-33,8)$ & $52,3(51,1-53,4)$ & $83,1(78,1-87,2)$ \\
\hline Privada & $13,0(12,1-14,1)$ & $37,4(35,5-39,5)$ & $49,5(47,4-51,7)$ & $16,9(12,8-21,9)$ \\
\hline \multicolumn{5}{|l|}{ Macrorregião * } \\
\hline Norte & $9,3(8,5-10,1)$ & $24,6(22,9-26,4)$ & $66,1(64,3-68,0)$ & $6,3(5,3-7,1)$ \\
\hline Nordeste & $10,3(12,5-15,2)$ & $37,3(35,4-39,2)$ & $48,9(46,7-51,2)$ & $20,8(19,1-22,7)$ \\
\hline Sudeste & $16,1(14,7-17,6)$ & $33,6(31,4-35,9)$ & $50,2(48,5-52,0)$ & $51,9(49,5-54,3)$ \\
\hline Sul & $16,9(15,7-18,1)$ & $33,9(31,9-35,9)$ & $49,2(46,9-51,5)$ & $11,8(10,6-13,1)$ \\
\hline Centro-oeste & $11,7(10,5-13,0)$ & $27,7(26,5-28,9)$ & $60,6(59,1-62,1)$ & $9,1(8,5-9,7)$ \\
\hline Total & $14,9(14,1-15,7)$ & $33,3(32,1-34,6)$ & $51,8(50,7-52,9)$ & \\
\hline
\end{tabular}

IC95\%: intervalo de 95\% de confiabilidade.

* Teste qui-quadrado $(p<0,05)$. 
Tabela 2

Caracterização da amostra com transtornos mentais comuns (TMC) de acordo com o tempo de aleitamento materno, segundo variáveis sociodemográficas, econômicas e geográficas entre escolares brasileiros de 12-17 anos. Estudo de Riscos Cardiovasculares em Adolescentes (ERICA), 2013-2014 ( $n=41.723)$.

\begin{tabular}{|c|c|c|c|c|c|c|}
\hline & \multicolumn{6}{|c|}{ Aleitamento materno (meses) } \\
\hline & \multicolumn{2}{|c|}{ Não ou $\leq 1$} & \multicolumn{2}{|c|}{$2-6$} & \multicolumn{2}{|c|}{$>6$} \\
\hline & TMC3 & TMC5 & TMC3 & TMC5 & TMC3 & TMC5 \\
\hline & \% (IC95\%) & $\%($ IC95\%) & $\%(I C 95 \%)$ & $\%($ IC95\%) & $\%(I C 95 \%)$ & $\%(I C 95 \%)$ \\
\hline \multicolumn{7}{|l|}{ Sexo } \\
\hline Feminino & $37,5(32,7-42,5)$ & $25,1(21,4-29,3)$ & $39,3(37,1-41,6)$ & $22,5(20,6-24,5)$ & $35,0(32,0-38,2)$ & $21,1(19,2-23,3)$ \\
\hline Masculino & $23,6(19,3-28,4)$ & $12,2(9,1-16,1)$ & $21,4(18,4-24,8)$ & $12,6(10,1-15,6)$ & $18,7(17,0-20,6)$ & $8,6(7,5-9,9)$ \\
\hline \multicolumn{7}{|l|}{ Idade (anos) } \\
\hline 12 & $28,4(19,2-39,8)$ & $20,6(11,9-33,3)$ & $24,3(19,5-29,7)$ & $13,6(9,0-20,0)$ & $19,2(16,5-22,4)$ & $9,0(7,1-11,3)$ \\
\hline 13 & $27,6(20,1-36,5)$ & $17,0(10,8-25,9)$ & $27,3(23,9-30,9)$ & $14,8(12,6-17,3)$ & $25,0(21,1-29,3)$ & $14,6(11,4-18,4)$ \\
\hline 14 & $29,1(23,4-35,5)$ & $17,3(12,6-23,3)$ & $33,2(28,8-38,0)$ & $20,1(17,2-23,4)$ & $27,2(23,1-31,8)$ & $15,3(12,7-18,4)$ \\
\hline 15 & $37,4(29,8-45,6)$ & $20,8(16,6-25,9)$ & $34,8(31,2-38,6)$ & $20,4(17,1-21,3)$ & $30,0(26,3-33,9)$ & $17,0(14,3-20,0)$ \\
\hline 16 & $31,3(23,0-41,1)$ & $19,5(13,7-26,8)$ & $37,0(33,5-40,6)$ & $21,5(18,1-25,3)$ & $33,4(31,0-36,0)$ & $18,3(16,4-20,4)$ \\
\hline 17 & $34,3(25,5-44,3)$ & $21,1(14,9-29,0)$ & $31,0(26,6-35,7)$ & $18,0(14,4-22,3)$ & $33,8(30,9-37,0)$ & $20,9(18,4-23,7)$ \\
\hline \multicolumn{7}{|l|}{ Cor da pele } \\
\hline Branca & $32,5(27,0-38,6)$ & $21,7(17,2-27,1)$ & $30,6(28,1-33,2)$ & $18,2(15,9-20,7)$ & $27,6(25,8-29,5)$ & $15,0(13,0-17,3)$ \\
\hline Preta & $31,2(20,8-44,0)$ & $12,6(8,2-19,0)$ & $31,9(25,4-39,3)$ & $19,3(14,2-25,8)$ & $31,5(27,2-36,1)$ & $18,5(15,3-22,2)$ \\
\hline Parda & $29,3(25,2-33,9)$ & $17,8(14,4-21,9)$ & $31,0(29,0-33,2)$ & $17,1(15,3-19,1)$ & $26,8(24,8-29,0)$ & $15,2(13,8-16,8)$ \\
\hline Amarela & $38,5(27,8-50,6)$ & $26,5(17,6-37,9)$ & $35,8(29,2-42,9)$ & $22,9(17,6-29,3)$ & $34,0(26,8-42,1)$ & $18,3(13,3-24,7)$ \\
\hline Indígena & $37,7(15,8-66,0)$ & $11,0(4,0-27,0)$ & $33,5(20,8-49,2)$ & $20,9(11,6-34,8)$ & $21,8(10,8-39,1)$ & $18,5(8,6-35,3)$ \\
\hline \multicolumn{7}{|l|}{ Classe econômica } \\
\hline A & $41,0(27,1-56,5)$ & $30,3(16,5-48,9)$ & $26,2(20,9-32,2)$ & $15,3(12,4-18,8)$ & $34,2(29,1-39,8)$ & $20,5(15,3-26,8)$ \\
\hline B & $31,9(26,6-37,7)$ & $19,4(14,6-25,2)$ & $31,6(28,2-35,2)$ & $18,5(15,9-21,5)$ & $26,3(24,6-27,1)$ & $13,8(12,3-15,6)$ \\
\hline $\mathrm{C}$ & $25,1(19,5-31,6)$ & $16,1(12,1-21,2)$ & $31,5(28,4-34,7)$ & $18,2(15,5-21,2)$ & $28,9(26,2-31,7)$ & $16,9(14,8-19,3)$ \\
\hline $\mathrm{D} / \mathrm{E}$ & $63,5(38,4-82,9)$ & $28,7(12,1-54,2)$ & $29,0(17,4-44,1)$ & $12,2(6,9-20,8)$ & $31,3(21,7-42,9)$ & $21,2(13,1-32,3)$ \\
\hline \multirow{2}{*}{\multicolumn{7}{|c|}{$\begin{array}{l}\text { Escolaridade } \\
\text { materna (anos) }\end{array}$}} \\
\hline & & & & & & \\
\hline$\geq 0$ e $<4$ & $27,7(19,8-37,2)$ & $16,1(10,8-23,4)$ & $33,2(27,7-39,3)$ & $18,3(14,3-23,1)$ & $25,8(20,1-32,5)$ & $12,5(9,5-16,1)$ \\
\hline$\geq 4 \mathrm{e}<8$ & $33,4(24,1-44,3)$ & $21,9(12,9-34,5)$ & $29,1(24,5-34,1)$ & $15,1(12,0-19,0)$ & $31,5(28,0-35,2)$ & $16,8(14,5-19,6)$ \\
\hline$\geq 8 \mathrm{e}<11$ & $31,9(24,2-40,7)$ & $19,8(13,3-28,5)$ & $32,5(28,5-36,8)$ & $19,8(16,5-23,6)$ & $26,5(23,3-20,9)$ & $15,7(13,3-18,4)$ \\
\hline $\begin{array}{l}\text { Ensino Médio } \\
\text { completo }\end{array}$ & $28,8(24,7-33,3)$ & $16,6(13,4-20,5)$ & $32,6(29,0-36,5)$ & $19,4(15,9-23,5)$ & $27,6(25,8-29,5)$ & $15,8(14,4-17,4)$ \\
\hline $\begin{array}{l}\text { Ensino Superior } \\
\text { completo }\end{array}$ & $29,3(19,8-41,1)$ & $29,3(19,8-41,1)$ & $25,0(21,6-28,7)$ & $14,8(12,4-17,6)$ & $27,0(24,4-29,7)$ & $15,1(13,0-17,5)$ \\
\hline \multicolumn{7}{|l|}{ Tipo de escola } \\
\hline Pública & $30,1(26,5-34,0)$ & $17,9(15,4-20,6)$ & $31,3(29,4-33,3)$ & $18,4(16,8-20,1)$ & $27,4(25,6-29,3)$ & $15,1(13,6-16,8)$ \\
\hline Privada & $37,8(30,4-45,9)$ & $27,7(18,9-38,6)$ & $30,0(27,7-32,4)$ & $16,1(12,6-20,3)$ & $29,0(26,9-31,2)$ & $17,3(15,8-18,8)$ \\
\hline \multicolumn{7}{|l|}{ Macrorregião } \\
\hline Norte & $35,5(31,1-40,2)$ & $20,9(16,9-25,5)$ & $32,9(29,7-36,3)$ & $19,0(16,6-21,7)$ & $30,1(28,2-32,2)$ & $17,2(15,7-18,8)$ \\
\hline Nordeste & $34,9(30,9-39,1)$ & $19,0(16,1-22,3)$ & $32,8(28,8-37,2)$ & $18,6(15,3-22,4)$ & $28,2(25,1-31,5)$ & $16,3(13,8-19,1)$ \\
\hline Sudeste & $29,1(29,5-35,4)$ & $19,1(14,9-24,1)$ & $30,0(27,8-32,3)$ & $17,5(15,3-19,9)$ & $26,1(23,5-28,7)$ & $14,2(11,9-16,8)$ \\
\hline Sul & $32,1(27,0-37,6)$ & $19,6(14,8-25,6)$ & $30,6(26,9-34,5)$ & $17,7(15,5-29,2)$ & $29,5(27,2-31,9)$ & $17,1(14,6-20,0)$ \\
\hline Centro-oeste & $34,2(29,3-39,4)$ & $20,8(16,4-25,9)$ & $32,4(30,1-34,9)$ & $18,6(16,3-21,1)$ & $30,5(28,5-32,6)$ & $17,2(15,6-18,9)$ \\
\hline
\end{tabular}

IC95\%: intervalo de 95\% de confiança. 
Tabela 3

Distribuição da prevalência de transtornos mentais comuns (TMC), cor da pele, escolaridade da mãe e macrorregião entre adolescentes com e sem informação sobre aleitamento materno. Estudo de Riscos Cardiovasculares em Adolescentes (ERICA), 2013-2014 ( $n=41.723)$.

\begin{tabular}{|c|c|c|}
\hline \multirow[t]{3}{*}{ Características } & \multicolumn{2}{|c|}{ Informação sobre aleitamento materno } \\
\hline & Sim (\%) & Não (\%) \\
\hline & {$[n=41.723]$} & {$[n=31.901]$} \\
\hline \multicolumn{3}{|l|}{ TMC } \\
\hline Sim & 29,3 & 30,9 \\
\hline \multicolumn{3}{|l|}{ Cor da pele } \\
\hline Branca & 40,0 & 40,0 \\
\hline Preta & 0,1 & 9,2 \\
\hline Parda & 49,7 & 47,7 \\
\hline Amarela & 2,0 & 2,3 \\
\hline Indígena & 0,6 & 0,8 \\
\hline \multicolumn{3}{|l|}{ Escolaridade materna (anos) } \\
\hline$\geq 0 \mathrm{e}<4$ & 13,6 & 19,1 \\
\hline$\geq 4 \mathrm{e}<8$ & 17,6 & 23,4 \\
\hline$\geq 8 \mathrm{e}<11$ & 20,7 & 18,0 \\
\hline Ensino Médio completo & 34,4 & 28,0 \\
\hline Ensino Superior completo & 13,5 & 9,6 \\
\hline \multicolumn{3}{|l|}{ Macrorregião } \\
\hline Norte & 7,0 & 11,4 \\
\hline Nordeste & 20,1 & 22,1 \\
\hline Sudeste & 51,9 & 49,1 \\
\hline Sul & 11,8 & 11,8 \\
\hline Centro-oeste & 9,1 & 5,6 \\
\hline Total & 56,7 & 43,3 \\
\hline
\end{tabular}

Observou-se uma tendência decrescente de prevalência de TMC em relação ao tempo de aleitamento materno, sendo maiores as prevalências entre os adolescentes que não foram amamentados quando comparados àqueles que receberam amamentação por mais de seis meses, tanto para TMC3 (31,2\% vs. $27,7 \%)$ quanto para TMC5 (19,3\% vs. 15,5\%), embora estas diferenças não tenham sido estatisticamente significativas (Tabela 5). Essa distribuição diferencial indica um possível efeito dose-resposta, independentemente do ponto de corte adotado. O modelo de regressão logística múltipla avaliando a associação entre aleitamento materno e TMC, ajustado simultaneamente pelas variáveis potencialmente de confundimento, identificou um efeito protetor da exposição mais prolongada ao aleitamento materno ( $>6$ meses), com uma redução estatisticamente significativa de $18 \%$ para TMC3 e de $26 \%$ para TMC5 (Tabela 5).

\section{Discussão}

Os adolescentes que receberam aleitamento materno por mais de seis meses mostraram-se menos propensos a serem identificados como casos de TMC para os dois pontos de corte do GHQ-12 analisados, mesmo após ajuste pelas variáveis de confundimento analisadas. Esses achados indicam um efeito protetor da exposição ao aleitamento materno prolongado sobre a saúde mental de adolescentes, ressaltando a importância da assistência materna e da formação de vínculos afetivos no início da vida 2,37 . 
Associação entre transtornos mentais comuns (TMC) e aleitamento materno, com razões de chances (OR) brutas e ajustadas por cada variável isoladamente, para os pontos de corte 3 e 5 no escore total do General Health Questionnaire versão 12 itens (GHQ-12), entre escolares brasileiros de 12-17 anos. Estudo de Riscos Cardiovasculares em Adolescentes (ERICA), 2013-2014 ( $n=41.723$ ).

\begin{tabular}{|c|c|c|}
\hline & \multicolumn{2}{|c|}{ TMC } \\
\hline & Ponto de corte $\geq 3$ & Ponto de corte $\geq 5$ \\
\hline & RI (IC95\%) & RI (IC95\%) \\
\hline \multicolumn{3}{|c|}{ Aleitamento materno (meses) } \\
\hline$\leq 1$ & 1,00 & 1,00 \\
\hline $2-6$ & $0,97(0,82-1,15)$ & $0,93(0,76-1,13)$ \\
\hline$>6$ & $0,83(0,72-0,96)$ * & $0,77(0,64-0,93)$ * \\
\hline \multicolumn{3}{|c|}{ Ajustado por cor da pele } \\
\hline \multicolumn{3}{|c|}{ Aleitamento (meses) } \\
\hline$\leq 1$ & 1,00 & 1,00 \\
\hline $2-6$ & $0,97(0,81-1,15)$ & $0,93(0,76-1,15)$ \\
\hline$>6$ & $0,82(0,71-0,95)$ * & $0,78(0,65-0,94)$ * \\
\hline \multicolumn{3}{|c|}{ Ajustado por macrorregião } \\
\hline \multicolumn{3}{|c|}{ Aleitamento (meses) } \\
\hline$\leq 1$ & 1,00 & 1,00 \\
\hline $2-6$ & $0,96(0,82-1,14)$ & $0,93(0,76-1,13)$ \\
\hline$>6$ & $0,82(0,71-0,94)$ * & $0,76(0,63-0,92)$ * \\
\hline \multicolumn{3}{|c|}{ Ajustado por escolaridade materna } \\
\hline \multicolumn{3}{|c|}{ Aleitamento (meses) } \\
\hline$\leq 1$ & 1,00 & 1,00 \\
\hline $2-6$ & $0,97(0,80-1,17)$ & $0,90(0,71-1,15)$ \\
\hline$>6$ & $0,83(0,70-0,98)$ & $0,74(0,60-0,92)$ * \\
\hline
\end{tabular}

IC95\%: intervalo de 95\% de confiança; RI: razão de incidência (modelos de regressão logística multivariada, com ajuste por cada variável isoladamente).

* $p<0,05$.

Existem poucos estudos que avaliam o efeito do aleitamento materno sobre transtornos mentais, alguns deles demonstraram um efeito benéfico do aleitamento materno sobre problemas de comportamento e bem-estar mental de crianças e adolescentes 11,13,14,15,16,18,38. No entanto, esses trabalhos diferem quanto ao desenho e categorização do tempo de aleitamento e, em alguns deles, foi estudado apenas o aleitamento materno exclusivo.

Em relação aos adolescentes, há um número ainda menor de estudos que avaliam a associação entre amamentação e saúde mental 14,18,26,38,39. Na maioria dos trabalhos, o efeito benéfico do aleitamento materno mostrou-se significativo quando realizado por períodos mais prolongados 14,18,38,39, corroborando os resultados deste estudo. Esse benefício provavelmente tem maior relação com o tempo de exposição ao leite materno do que em função do padrão da amamentação (exclusivo ou não).

Uma via possível de explicação de associação entre aleitamento materno e saúde mental poderia ser a composição do leite materno, rico em ácidos graxos essenciais, que são elementos fundamentais para o desenvolvimento cerebral 40,41,42,43. A exposição a esses nutrientes parece estar relacionada a maiores níveis de habilidades cognitivas, medidas por testes que avaliam o quociente de inteligência (QI) em crianças, tendo sido encontrada uma associação entre baixo QI e depressão 40,41,42,43.

E o ambiente doméstico e/ou o cuidado materno na infância, direcionando suas explicações à afetividade proporcionada pela interação mãe/filho durante a amamentação, momento no qual a criança experimenta sensações semelhantes às vividas no período intrauterino, acalmando e fortalecendo os laços mãe/filho 44,45,46. 
Prevalência de transtornos mentais comuns (TMC) e associação com aleitamento materno para os pontos de corte 3 e 5 no escore total do General Health Questionnaire versão 12 itens (GHQ-12), entre escolares brasileiros de 12-17 anos. Estudo de Riscos Cardiovasculares em Adolescentes (ERICA), 2013-2014 ( $\mathrm{n}=41.723$ ).

\begin{tabular}{|c|c|c|c|c|}
\hline & \multicolumn{4}{|c|}{ TMC } \\
\hline & \multicolumn{2}{|c|}{ Ponto de corte $\geq 3$} & \multicolumn{2}{|c|}{ Ponto de corte $\geq 5$} \\
\hline & RI (IC95\%) & $\%($ IC95\%) & RI (IC95\%) & $\%($ IC95\%) \\
\hline Amostra total & & $29,3(28,1-30,5)$ & & $16,9(15,9-17,9)$ \\
\hline \multicolumn{5}{|c|}{ Aleitamento materno (meses) } \\
\hline$\leq 1$ mês & 1,00 & $31,2(27,7-35,0)$ & 1,00 & $19,3(16,7-22,3)$ \\
\hline $2-6$ & $0,96(0,80-1,15)$ & $31,0(29,4-32,7)$ & $0,89(0,70-1,13)$ & $17,9(16,5-19,5)$ \\
\hline$>6$ & $0,82(0,69-0,97)$ * & $27,7(26,1-29,2)$ & $0,74(0,59-0,91)$ * & $15,5(14,1-16,9)$ \\
\hline
\end{tabular}

IC95\%: intervalo de 95\% de confiança; RI: razão de incidência (modelos de regressão logística múltipla, com ajuste por cor da pele, macrorregião e escolaridade materna).

* $\mathrm{p}<0,05$.

Entretanto, neste estudo, não foi possível obter informações sobre as características do ambiente doméstico durante a infância nem sobre a ligação materno-infantil, portanto, não podemos avaliar se o efeito protetor do aleitamento materno sobre a saúde mental é atribuível aos componentes do leite, à ligação mãe/filho ou a uma combinação de ambos.

Embora a associação entre aleitamento materno e saúde mental tenha se mantido após ajustes por cor da pele, escolaridade da mãe e região de residência, em ambos os pontos de cortes, o efeito protetor da amamentação prolongada foi mais forte para TMC5, indicando que um número maior de sintomas para a classificação de TMC aumenta as chances desses indivíduos serem casos verdadeiro-positivos, reduzindo um erro não diferencial que pode levar à subestimação da associação.

É importante considerar que o GHQ-12 é um instrumento de rastreamento de sintomas presentes nas duas semanas anteriores à entrevista, e que alterações psicológicas recentes podem influenciar as respostas dos adolescentes 30 . Além disso, os sintomas de TMC em crianças e adolescentes podem ser manifestações iniciais e menos específicas de transtornos mentais mais graves, que somente serão detectados em fases posteriores 30,37 . Nesse sentido, o rastreamento de TMC em crianças e adolescentes pode ser um procedimento de extrema valia para a identificação precoce do risco de adoecimento mental subsequente e indicação da necessidade de seguimento. Por fim, considerando que tenha havido um possível viés de classificação devido à pouca acurácia do GHQ, a introdução de erro não diferencial, isto é, o mesmo para expostos (amamentados) e não expostos (não amamentados), levaria a uma atenuação da associação. É possível, portanto, que as associações entre a exposição ao aleitamento materno e a ocorrência de TMC sejam ainda de maior magnitude do que as encontradas neste estudo e, portanto, conferindo maior efeito protetor.

Resultados semelhantes em relação a características sociodemográficas e econômicas também foram encontrados em outros estudos 11,30,47,48,49,50. A maior prevalência de TMC entre os adolescentes do sexo feminino e entre adolescentes mais velhos é corroborada por outros trabalhos que também avaliaram a distribuição desses transtornos por sexo e idade, mesmo com a utilização de diferentes instrumentos de coleta 48,49,50,51. Na amostra total do estudo ERICA, da qual esta subamostra faz parte, também foi identificada uma maior prevalência de TMC entre meninas e adolescentes de 15-17 anos 30 , o que corrobora a representatividade da subamostra estudada.

A elevada prevalência de TMC entre crianças e adolescentes é preocupante, pois estes transtornos tendem a influenciar negativamente o seu pleno desenvolvimento emocional, educacional e o potencial para que vivam vidas satisfatórias e produtivas, além de serem potencialmente persistentes, fazendo com que uma parcela importante destes indivíduos apresente-se mais tarde como casos graves de transtornos mentais, causando comprometimento em sua vida social, como por exemplo, estabelecer-se no mercado de trabalho, construção de laços afetivos e aumento à exposição à violência (seja como 
autor ou como vítima) na vida adulta 1,52. Aplicando-se as estimativas de prevalência encontradas neste estudo à estrutura populacional do Censo Demográfico de 2010, haveria o acometimento de 3,4 a 6 milhões de adolescentes brasileiros 53 .

Vale ainda ressaltar que mais de $50 \%$ dos adolescentes foram amamentados na infância por um tempo maior que seis meses, sendo o Norte a região onde o aleitamento materno prolongado foi mais prevalente. Essa informação reforça a ideia de que o tempo de duração do aleitamento materno no Brasil vem aumentando 54 . Na década de 1970, as crianças brasileiras eram amamentadas, em média, por dois meses e meio, chegando a um ano e dois meses em 2006/2007 54. Outros estudos também identificaram maiores taxas de aleitamento materno por períodos mais longos na Região Norte, o que pode ser um indicativo de que, em regiões de maior pobreza, o aleitamento materno é a principal fonte de alimentação para os recém-nascidos 54,55.

Entre as limitações deste estudo, pode-se considerar que a informação acerca da exposição ao aleitamento materno é suscetível a vieses de memória, embora possa ser considerada relativamente precisa devido à sua relevância no histórico dos ciclos vitais; além disso, não haveria uma justificativa plausível para que houvesse uma distribuição diferencial desse recordatório materno em função da ocorrência de TMC nos adolescentes. Por outro lado, é possível que as mães tendam a superestimar o tempo de aleitamento, considerando a expectativa de cumprimento dos papéis maternos na sociedade, mas também não se esperaria uma distribuição diferencial entre os estratos. Este trabalho avaliou a presença de sintomas indicativos de TMC somente nas duas últimas semanas anteriores à entrevista, o que pode ter deixado de identificar adolescentes que já apresentaram estes sintomas em períodos pregressos, produzindo uma subestimativa da prevalência de TMC na população estudada. Além disso, este estudo avaliou somente os adolescentes escolares em cidades com mais de 100 mil habitantes, não sendo possível generalizar os achados para os adolescentes não escolares, possivelmente em situação de maior risco social e de maior vulnerabilidade. É provável que, nessas populações, tanto o aleitamento materno seja menos frequente e menos prolongado quanto a ocorrência de TMC seja maior. Por fim, vale lembrar que este trabalho avaliou somente a presença de TMC, não incluindo a identificação de transtornos mentais mais graves e incapacitantes, que podem ter contribuído tanto para a inelegibilidade quanto para a exclusão da participação neste estudo, como psicoses, transtornos do espectro autista, retardo mental e dependência de substâncias psicoativas, condições que também estão frequentemente associadas a maiores taxas de evasão escolar e à incapacitação decorrente.

Apesar da implantação de novas políticas públicas voltadas para a atenção em saúde mental de crianças e adolescentes nos últimos anos, com a construção de redes ampliadas e intersetoriais e a oferta de Centros de Atenção Psicossocial Infanto-juvenil (CAPSi), o cuidado desse público continua sendo um desafio no país, tanto pela escassez como pela distribuição desigual destes serviços entre as unidades federativas e regiões 56,57. Além disso, esses programas visam ao atendimento de crianças e adolescentes que apresentam prioritariamente intenso sofrimento psíquico decorrente de transtornos mentais graves e persistentes, não contemplando a atenção a crianças e adolescentes que apresentam TMC.

\section{Conclusões e recomendações}

Este trabalho evidenciou um efeito protetor do aleitamento materno prolongado para a saúde mental de adolescentes, enfatizando a importância do cuidado e da formação de vínculos afetivos no início da vida, e fortalecendo a necessidade de implantação e manutenção de políticas públicas que valorizem e estimulem o aleitamento materno prolongado. Além disso, sendo elevada a prevalência de TMC entre adolescentes e considerando a sua persistência e potencial de produzir incapacitação, é premente a necessidade de promover medidas e programas de promoção de saúde mental na população em geral, da organização de sistemas de vigilância para a identificação precoce e seguimento dos casos, além da ampliação da rede de serviços de saúde mental para assistir a essa população nas diversas regiões do Brasil. 


\section{Colaboradores}

C. R. Almeida contribuiu na concepção e planejamento do estudo, análise e interpretação dos dados, revisão bibliográfica, discussão dos resultados, redação do manuscrito. E. S. F. Coutinho e K. V. Bloch contribuíram na análise e interpretação dos dados, discussão dos resultados. D. A. Silva colaborou na análise e interpretação dos dados, discussão dos resultados, redação do manuscrito. E. R. A. Oliveira contribuiu na concepção e planejamento do estudo. M. C. Viana contribui na concepção e planejamento do estudo, análise e interpretação dos dados, discussão dos resultados, redação do manuscrito. Todos os autores revisaram e aprovaram a versão final do artigo.

\section{Informações adicionais}

ORCID: Caroline Rodrigues de Almeida (00000001-5853-7482); Evandro Silva Freire Coutinho (0000-0002-4649-7353); Daniela Alves Silva (00000001-7396-2305); Elizabete Regina Araújo de Oliveira (0000-0002-6616-4273); Katia Vergetti Bloch (0000-0002-6992-3159); Maria Carmen Viana (0000-0002-0464-4845).

\section{Agradecimentos}

Aos pesquisadores e coordenação do ERICA.

\section{Referências}

1. World Health Organization. Child and adolescent mental health 2016. Geneva: World Health Organization; 2016.

2. Garrison CZ, Jackson KL, Marsteller F, McKeown R, Addy C. A longitudinal study of depressive symptomatology in young adolescents. J Am Acad Child Adolesc Psychiatry 1990; 29:581-5.

3. Benjet C, Borges G, Méndez E, Albor Y, Casanova L, Orozco R, et al. Eight-year incidence of psychiatric disorders and service use from adolescence to early adulthood: longitudinal follow-up of the Mexican Adolescent Mental Health Survey. Eur Child Adolesc Psychiatry 2016; 25:163-73.

4. Robinson M, Oddy WH, Li J, Kendall GE, de Klerk NH, Silburn SR, et al. Pre- and postnatal influences on preschool mental health: a largescale cohort study. J Child Psychol Psychiatry 2008; 49:1118-28.

5. Thiengo DM, Cavalcante MT, Lovisi GM. Prevalência de transtornos mentais entre crianças e adolescentes e fatores associados: uma revisão sistemática. J Bras Psiquiatr 2014; 63: 360-72.

6. Associação Brasileira de Psiquiatria. Pesquisa sobre sintomas de transtornos mentais e utilização de serviços em crianças brasileiras de 6 a 17 anos. http://www.abpbrasil.org.br/me dicos/pesquisas/ (acessado em 05/Jul/2017).

7. Conceição TV. Crianças e adolescentes vulneráveis: o atendimento interdisciplinar nos centros de atenção psicossocial. Rev Bras Psiquiatr 2011; 33:420-1.

8. Campestrini S. Tecnologia simplificada na enfermagem materno infantil. Rev Bras Enferm 1991; 44:53-63.

9. Santos JS, Andrade M, Silva JL. Fatores que influenciam no desmame precoce: implicações para o enfermeiro de promoção da saúde na estratégia de saúde da família. Informe-se 2009; 5:26-9.

10. Horta BL, Loret de Mola C, Victora CG. Breastfeeding and intelligence: systematic review and meta-analysis. Acta Paediatr 2015; 104:14-9.

11. Taylor B, Wadsworth J. Breast feeding and child development at five years. Dev Med Child Neurol 1984; 26:73-80.

12. Victora CG, Horta BL, Loret de Mola C, Quevedo L, Pinheiro RT, Gigante DP, et al. Association between breastfeeding and intelligence, educational attainment, and income at 30 years of age: a prospective birth cohort study from Brazil. Lancet Glob Health 2015; 3:e199205.

13. Heikkila K, Sacker A, Kelly Y, Renfrew MJ, Quigley MA. Breastfeeding and child behaviour in the Millennium cohort study. Arch Dis Child 2011; 96:635-42.

14. Hayatbakhsh MRO, Callaghan MJ, Bor W, Williams GM, Najman JM. Association of breastfeeding and adolescents' psychopathology: a large prospective study. Breastfeed Med 2012; 7:480-6. 
15. Liu F, Ma LJ, Yi MJ. Association of breastfeeding with behavioral problems and temperament development in children aged 4-5 years. Zhongguo Dang Dai Er Ke Za Zhi 2006; 8:334-7.

16. Yi MJ, Zhou XB, Zhang P, Liu XM. Correlation of behavioral problems with gender and infant breastfeeding in pre-school children. Chinese Journal of Clinical Rehabilitation 2005; 9:2435.

17. Montgomery SM, Ehlin A, Sacker A. Breastfeeding and resilience against psychosocial stress. Arch Dis Child 2006; 91:990-4.

18. Oddy WH, Kendall GE, Li J, Jacoby P, Robinson $\mathrm{M}$, Klerk $\mathrm{NH}$, et al. The long-term effects of breastfeeding on child and adolescent mental health: a pregnancy cohort study followed for 14 years. J Pediatr 2010; 156:568-74

19. Reynolds D, Hennessy E, Polek E. Is breastfeeding in infancy predictive of child mental well-being and protective against obesity at 9 years of age? Child Care Health Dev 2014; 40:882-90.

20. Allen NB, Lewinsohn PM, Seeley JR. Prenatal and perinatal influences on risk for psycho pathology in childhood and adolescence. Dev Psychopathol 1998; 10:513-29.

21. Peus V, Redelin E, Scharnholz B, Paul T, Gass $\mathrm{P}$, Deuschle P, et al. Breast-feeding in infancy and major depression in adulthood: a retrospective analysis. Psychother Psychosom 2012; 81:189-90.

22. Mimouni-Bloch A, Kachevanskaya A, Mimouni FB, Shuper A, Raveh E, Linder N. Breastfeeding may protect from developing attention deficit and hyperactivity disorder. Eur J Paediatr Neurol 2013; 17 Suppl 1:S96.

23. Stadler DD, Musser ED, Holton KF, Shannon J, Nigg JT. Recalled initiation and duration of maternal breastfeeding among children with and without ADHD in a well characterized case-control sample. J Abnorm Child Psychol 2016; 44:347-55.

24. Anselmi, L. Barros FC, Minten GC, Gigante DP, Horta BL, Victora CG. Prevalence and early determinants of common mental disorders in the 1982 birth cohort, Pelotas, Southern Brazil. Rev Saúde Pública 2008; 42 Suppl 2:2633.

25. Kramer MS, Matush L, Bogdanovich N, Aboud F, Mazer B, Fombonne E, et al. Health and development outcomes in 6.5-y-old children breastfed exclusively for 3 or 6 mo. Am J Clin Nutr 2009; 90:1070-4.

26. Kwok MK, Leung GM, Schooling CM. Breastfeeding and early adolescent behaviour, selfesteem and depression: Hong Kong's 'Children of 1997' birth cohort. Arch Dis Child 2013; 98:887-94.

27. Lind JN, Li R, Perrine CG, Schieve LA. Breastfeeding and later psychosocial development of children at 6 years of age. Pediatrics 2014; 134 Suppl 1:S36-41.

28. Goldberg D, Huxley P. Common mental disorders: a bio-social model. London: Tavistock; 1992.
29. Figueiredo VC, Szklo AS, Costa LC, Kuschnir MCC, Da Silva TLN, Bloch KV, et al. ERICA: prevalência de tabagismo em adolescentes brasileiros. Rev Saúde Pública 2016; 50 Suppl $1: 12 \mathrm{~s}$.

30. Lopes CS, Abreu GDA, Santos DF, Menezes PR, Carvalho KMB, Cunha CDF, et al. Prevalência de transtornos mentais comuns em adolescentes brasileiros. Rev Saúde Pública 2016; 50 Suppl 1:14s.

31. Wan Y-P, Sá Junior AR. Questionário de saúde geral de Goldberg (qsg). Intrumentos de avaliação em saúde mental. Porto Alegre: Artmed; 2016.

32. Bloch KV, Cardoso MA, Sichieri R. Estudo dos Riscos Cardiovasculares em Adolescentes (ERICA): resultados e potencialidade. Rev Saúde Pública 2016; 50 Suppl 1:2s.

33. Associação Brasileira de Empresas de Pesquisa. Critério Brasil 2015 e atualização da distribuição de classes para 2016. São Paulo: Associação Brasileira de Empresas de Pesquisa; 2016.

34. Pearce N. Effect measures in prevalence studies. Environ Health Perspect 2004; 112:104750.

35. Reichenheim ME, Coutinho ES. Measures and models for causal inference in cross-sectional studies: arguments for the appropriateness of the prevalence odds ratio and related logistic regression. BMC Med Res Methodol 2010; 10:66.

36. Vasconcellos MTL, Silva PLN, Szklo M, Kuschnir MCC, Klein CH, Abreu GA, et al. Sampling design for the Study of Cardiovascular Risk in Adolescents (ERICA). Cad Saúde Pública 2015; 31:921-30.

37. Cid MFB, Matsukura TS, Silva MDP. Transtorno mental materno e desenvolvimento infantil: percepções sobre essa realidade. Mundo Saúde (Impr.) 2012; 36:265-75.

38. Mola C, Horta BL, Gonçalves H, Quevedo LDA. Breastfeeding and mental health in adulthood: A birth cohort study in Brazil. J Affect Disord 2016; 202:115-19.

39. Cable N, Bartley M, McMunn A, Kelly Y. Gender differences in the effect of breastfeeding on adult psychological well-being. Eur J Public Health 2012; 22:653-8.

40. Crawford MA. The role of essential fatty acids in neural development: implications for perinatal nutrition. Am J Clin Nutr 1993; 57(5 Suppl):703S-9S.

41. Birch EE, Garfield S, Castaneda Y, HughbanksWheaton D, Uauy R, Hoffman D. Visual acuity and cognitive outcomes at 4 years of age in a double-blind, randomized trial of long-chain polyunsaturated fatty acid-supplemented infant formula. Early Hum Dev 2007; 83:279-84.

42. Sinn N, Milte C, Howe PR. Oiling the brain: a review of randomized controlled trials of omega- 3 fatty acids in psychopathology across the lifespan. Nutrients 2010; 2:128-70. 
43. Bjerve KS, Brubakk AM, Fougner KJ, Johnsen $\mathrm{H}$, Midthjell K, Vik T. Omega-3 fatty acids: essential fatty acids with important biological effects, and serum phospholipid fatty acids as markers of dietary omega 3-fatty acid intake. Am J Clin Nutr 1993; 57(5 Suppl):801S-5S.

44. Gauthier L, Stollak G, Messe L, Aronoff J. Recall of childhood neglect and physical abuse as differential predictors of current psychological functioning. Child Abuse Negl 1996; 20:54959.

45. Rey JM. Perceptions of poor maternal care are associated with adolescent depression. J Affect Disord 1995; 34:95-100.

46. Moore ER, Bergman N, Anderson GC, Medley N. Early skin-to-skin contact for mothers and their healthy newborn infants. Cochrane Database Syst Rev 2012; (5):CD003519.

47. Pinheiro KAT, Horta BL, Pinheiro RT, Horta LL, Terres NG, Silva RA. Common mental disorders in adolescents: a population based cross-sectional study. Rev Bras Psiquiatr 2007; 29:241-5.

48. Jansen K, Mondin TC, Ores LC, Souza LDM, Konradt CE, Pinheiro RT, et al. Transtornos mentais comuns e qualidade de vida em jovens: uma amostra populacional de Pelotas, Rio Grande do Sul, Brasil. Cad Saúde Pública 2011;27:440-8.

49. Silva RS, Costa LA. Prevalência de transtornos mentais comuns entre estudantes universitários da área da saúde. Encontro: Revista de Psicologia 2012; 15:105-12.

50. Coutinho LMS, Matijasevich A, Scazufca M, Menezes PR. Prevalência de transtornos mentais comuns e contexto social: análise multinível do São Paulo Ageing er Health Study (SPAH). Cad Saúde Pública 2014; 30:1875-83.
51. Valadares C. Brasil é referência mundial em amamentação. Agência Saúde. http://dab. saude.gov.br/portaldab/noticias.php?conteu do=_\&cod=2223 (acessado em 05/Jul/2017).

52. Patel V, Flisher AJ, Hetrick S, McGorry P. Mental health of young people: a global publichealth challenge. Lancet 2007; 369:1302-13.

53. Instituto Brasileiro de Geografia e Estatística. Censo demográfico 2010. ftp://ftp.ibge.gov. br/Indicadores_Sociais/Criancas_e_Adoles centes/1997/Caracteristicas_Gerais/ (acessado em 02/Fev/2018).

54. Wenzel D, Souza SB. Prevalência do aleitamento materno no Brasil segundo condições socioeconômicas e demográficas. Rev Bras Crescimento Desenvolv Hum 2011; 21:251-8.

55. Venancio SI, Escuder ML, Saldiva ARDM, Giugliani ERJ. Breastfeeding practice in the Brazilian capital cities and the Federal District: current status and advances. J Pediatr (Rio J.) 2010; 86:317-24.

56. Couto MCV, Delgado PGG. Crianças e adolescentes na agenda política da saúde mental brasileira: inclusão tardia, desafios atuais. Psicol Clín 2015; 27:17-40.

57. Assis SG, Avanci JQ, Pesce RP, Ximenes LF. Situação de crianças e adolescentes brasileiros em relação à saúde mental e à violência. Ciênc Saúde Colet 2009; 14:349-61. 


\section{Abstract}

This article sought to evaluate the effect of exposure, and exposure time, to breastfeeding on the occurrence of common mental disorders (CMD) among Brazilian adolescents enrolled in school. This study analyzed data from the Study of Cardiovascular Risk in Adolescents (ERICA), evaluating those whose questionnaire regarding breastfeeding had been filled out by parents or guardians. The presence of CMD was identified using the General Health Questionnaire, version 12 items (GHQ-12), and we considered two cutoff points (scores $\geq 3$ and $\geq 5$ ). We tested the associations in bivariate analyses and through multiple logistical regression models, adjusting for potential confounding variables. Of the 41,723 adolescents we evaluated, most were students of the female sex (54.6\%), aged between 12 and 15 years (71\%), attended public schools (83.1\%), resided in the Southeastern region (51.9\%) and belonged to the economic classes B (53.8\%) and C (34.1\%). Around half of the adolescents' mothers had not completed their secondary education (51.7\%). The group of adolescents who were breastfed for more than six months (51.8\%) had a lower CMD prevalence for both GHQ-12 cutoff points, when compared with the group who were not breastfed or who were breastfed for $\leq 1$ month $(I R=0.82$; 95\%CI: 0.69-0.97 and IR = 0.74; 95\%CI: 0.590.91 for 3 and 5 points, respectively). Prolonged breastfeeding seems to play a protective role on the occurrence of CMD in adolescence.

Mental Health; Adolescent; Mental Disorders; Breast Feeding

\section{Resumen}

El objetivo fue evaluar el efecto de la exposición $y$ del tiempo de exposición a la lactancia materna en la ocurrencia de trastornos mentales comunes (TMC) entre adolescentes escolares brasileñas. Este trabajo analizó datos del Estudio de Riesgos Cardiovasculares en Adolescentes (ERICA), evaluando aquellos, cuyo cuestionario -referente a la lactancia materna-habia sido respondido por los padres o responsables legales. La presencia de TMC fue identificada por el General Health Questionnaire, versión 12 items (GHQ-12), considerándose dos puntos de corte (puntuaciones $\geq 3 y \geq 5$ ). Las asociaciones fueron probadas en análisis bivariados, y a través de modelos de regresión logística múltiple, con ajustes por variables potenciales de confusión. Entre los 41.723 adolescentes evaluados, la mayoría estuvo compuesta por estudiantes que eran del sexo femenino $(54,6 \%)$, que tenían una edad entre 12 y 15 años (71\%), estudiaban en escuelas públicas $(83,1 \%)$, residían en la región Sudeste $(51,9 \%)$ y eran de las clases económicas $B(53,8 \%)$ y $C(34,1 \%)$. Cerca de la mitad de las madres de los adolescentes no contaba con la enseñanza media completa (51,7\%). El grupo de adolescentes con más de seis meses de lactancia materna $(51,8 \%)$ presentó una menor prevalencia de TMC para los dos puntos de corte del GHQ12 evaluados, cuando se comparan con el grupo que no recibió lactancia materna o que la recibió durante un período $\leq 1$ mes $(R I=0,82$; IC95\%: 0,69-0,97 y $R I=0,74$ y IC95\%: 0,59-0,91 para $3 y$ 5 puntos, respectivamente). La lactancia materna prolongada parece desempeñar un papel protector para la ocurrencia de TMC en la adolescencia.

Salud Mental; Adolescente; Trastornos Mentales; Lactancia Materna
Recebido em 21/Mai/2018

Versão final reapresentada em 25/Nov/2018 Aprovado em 10/Dez/2018 